\title{
Wird der Vorstand \\ zum Regierungssprecher \\ seines Computers? ${ }^{1}$
}

von Dieter Bartmann

Otto-Friedrich-Universität Bamberg

\begin{tabular}{|ll|}
\hline 1 & Macht durch Computer \\
2 & Machtübernahme der Computer? \\
3 & Die beiden Wissensquellen des Computers \\
4 & Stärken und Schwächen von Anwendungssystemen \\
5 & Beseitigung des Methodendefizits \\
6 & Zielsetzung Basisprobleme \\
7 & Konklusion \\
\hline
\end{tabular}

\section{Zusammenfassung}

Besteht die Möglichkeit, daß sich mit zunehmender Leistungsfähigkeit die Computersysteme zu einer Bedrohung für das Topmanagement entwickeln? Wird in ferner Zukunft der Unternehmensvorstand zum Regierungssprecher seines eigenen Computers? Um diese Fragen ranken sich die nachfolgenden Überlegungen im ersten Teil des Aufsatzes. Es werden Stärken und Schwächen von Anwendungssystemen untersucht. Im zweiten Teil wird auf das Methodendefizit hingewiesen. Die Konsequenz heißt: Methodeninnovation zur Lösung von Problemen an der Unternehmensbasis. Es werden zwei Beispiele angeführt, wie man durch Wissensimport in Form von Methodeninnovationen auf Werkstattebene das Betriebsergebnis spürbar verbessern kann.

\section{Macht durch Computer}

Wissen gründet sich auf Information. Gemäß dem Sprichwort „Wissen ist Macht“ müßte ein computergestütztes Informations- und Planungssystem zu einem deutlichen Machtzuwachs des Unternehmens führen. Stimmt das?

Die ersten Anwendungen der kommerziellen Datenverarbeitung betrafen die Massendatenverarbeitung. Es wurden Tätigkeiten automatisiert, die man bislang sehr

\footnotetext{
${ }^{1}$ Für eine anregende Diskussion und die griffige Formulierung des Themas danke ich meinem Kollegen, dem Philosophen Walther Zimmerli.
} 
mühsam per Hand durchführen mußte. Der DV-Einsatz erstreckte sich auf einer relativ niedrigen Ebene innerhalb der Unternehmenshierarchie. Nach und nach wurde sie dann zur Lösung von Problemen auf höheren Unternehmensebenen eingesetzt. Zeichnet man den Kurvenverlauf, wie in Abhängigkeit von der Zeit die Datenverarbeitung zur Problemlösung in den verschiedenen Unternehmensebenen herangezogen wurde, erhält man eine typische $S$-Kurve. In der "Community of the Leading Horses" beginnt sich die Kurve zunehmend abzuflachen. Dort ist der Durchdringungsgrad am höchsten und die DV-Stärke am weitesten entwickelt.

Unter DV-Stärke wird

- zum einen der Grad der Verfügbarkeit aktueller Informationen über die IstZustände des Unternehmens,

- zum anderen das Maß der Zuverlässigkeit dieser Daten,

- zum dritten Umfang und Qualität der Abbildung der Dynamik von Unternehmensvorgängen auf den Computer und

- zum vierten die Fähigkeit zur DV-gestützten kostenorientierten Steuerung bei möglichst geringem Zeitverzug

verstanden.

Die DV-Stärke ist zu einem wichtigen Wettbewerbsfaktor geworden. Innerbetrieblich ist sie sowohl ein Produktionsfaktor als auch ein Inputfaktor für Produktinnovationen. Nach außen wird sie als strategische Waffe eingesetzt.

Innovationsfähigkeit bedeutet die Fähigkeit, technische Neuerungen zu entdecken oder $\mathrm{zu}$ entwickeln und gewinnbringend $\mathrm{zu}$ nutzen. Letzteres ist entscheidend vom Gestaltungsspielraum und der „Beschleunigungskraft“ des Unternehmens, das heißt der Änderungsgeschwindigkeit zur Ansteuerung des Ziels „Innovation bei niedrigen Kosten", und damit von der DV-Stärke abhängig.

Der Einsatz der Datenverarbeitung als strategische Waffe dient dem Aufbau einer Gemeinschaft Anbieter - Kunden zu dem Zweck, den Kunden durch die Realisierung von Synergieeffekten ins gemeinsame Boot zu locken und gleichzeitig durch die Erhöhung der Austrittsbarrieren an sich zu binden. Dies kann durch die Errichtung betriebsübergreifender Informations- und Vorgangsketten geschehen, durch Hardwareausstattung und durch Übertragung von computerintegrierten Organisationsformen auf den Kunden ( $z$. B. durch das Zuverfügungstellen von Programmen). Näheres hierzu findet der Leser in [3].

\section{Machtübernahme der Computer?}

Spätestens bei der Realisierung des Computer Integrated Manufacturing ist die Integration von Computeranwendungssystemen in die gesamtbetrieblichen Abläufe so hoch, daß es nicht mehr möglich ist, eventuell etwas weniger Computereinsatz mit etwas mehr Organisationskunst und personellem Planungswissen zu kompensieren. Die Planungs- und Steuerungsfähigkeit eines Unternehmens ist zu einer resultierenden Größe geworden, deren unabhängige Variable die DV-Stärke ist. 
Computersysteme unterstützen aber nicht nur. Dadurch, daß jede Interaktion zwischen Mensch und Maschine seine Datenspuren hinterläßt, wird sie aktenkundig. Bei den Management Informationssystemen (MIS) der zukünftigen Generation ist dies nicht nur ein Nebeneffekt, sondern eine Hauptfunktion. Der Topmanager baut sich ein Netzwerk von Beziehungen zu Kunden, Lieferanten, Aufsichtsräten, Kollegen, Mitarbeitern, Banken, Universitäten etc. auf und verwendet einen erheblichen Teil seiner Aktivitäten mit der Pflege, Erweiterung und Bereinigung dieser Kontakte. Der „networking“ Manager wird das Leitbild der Managementunterstützung sein und die MIS-Forschung wird ihre Hauptaufgabe in der Entwicklung von Konzepten und Systemen für das „Management Information Networking“ (MIN) sehen ([1]). Das Beziehungsgeflecht (verknüpft durch Gespräche, Briefe, Arbeitskreise, Delegation von Aufgaben, Durchführung von Kontrollaufgaben, Anforderung von Supports) wird im Computer in Form eines Diagrammes gespeichert. Es läßt Defizite und Stärken eines Managers deutlich erkennen (z. B. Stabilität und Offenheit eines Netzes, mangelnde starke Verbindungen).

Nicht zuletzt aufgrund dieser Entwicklung, die als Bedrohung empfunden werden kann, und auch wegen der Fachfremdheit stehen manche Topmanager dieser neuen Technologie teilweise etwas reserviert und verunsichert gegenüber. Haben sie früher als Chefpiloten das Unternehmensflugzeug mit Unterstützung weniger Instrumente mehr oder weniger auf Sicht gesteuert, so sind sie jetzt in das moderne Cockpit eines Großraumflugzeuges versetzt, wo eine erschreckende Anzahl von Instrumenten, Kontrollampen und Frühwarnsystemen den gesamten Steuerungsprozeß äußerst komplex erscheinen läßt, und außerdem wichtige Teilfunktionen der menschlichen Kontrolle entzogen sind und vollständig automatisiert ablaufen. So ist es verständlich, wenn manche Vorstände und Geschäftsführer am Horizont die Gefahr heraufdämmern sehen, daß mit der Übernahme von Steuerungsfunktionen das DV-System auch in ihrem Aufgabenbereich schrittweise Zuständigkeiten übernimmt. Im ersten Schritt werden die Fakten entweder elementar oder verdichtet in einer gewünschten $\mathrm{Be}$ richtsform präsentiert (Reportsysteme, Planungssprachen). Im zweiten Schritt werden Entscheidungsrahmen von disponiblen Größen mitgeliefert. Im dritten Schritt werden die Entscheidungen selbst vorgeschlagen (Decision Support Systems, Expertensysteme) und im vierten Schritt quantitativ so überzeugend begründet, daß die Vorschläge schon fast die Form von Sachzwängen annehmen.

Wird im Endstadium die Unternehmensleitung zum Regierungssprecher des eigenen Computers degradiert?

Die spontane Reaktion auf diese provozierende Frage lautet: hoffentlich nicht. Und es lassen sich sofort zwei Gründe dafür anführen.

Erstens gibt es viele Probleme auf Vorstandsebene, die sich einer strengen Betrachtung entziehen. Ist zum Beispiel die Entscheidung über die Errichtung eines Zweigwerkes zu treffen, dann gilt es, die vordergründig betriebswirtschaftlichen Ziele mit den regional- und sozialpolitischen Gesichtspunkten zu harmonisieren. Man kann derartige Probleme nur ungenügend quantifizieren und rationalen Methoden zugänglich machen (obwohl in der betriebswirtschaftlichen Theorie auch hierzu Konzepte 
im Rahmen des Multi Criteria Decision Making entwickelt wurden), sondern man braucht zu ihrer Lösung das gewisse Gespühr. Gerade das zeichnet den guten Topmanager aus.

Derartige Probleme wird es immer geben. Jedoch ist ein deutlicher Trend zu sehen: Was früher noch nebelhaft verschwommen war, versucht man heutzutage analytisch zu erhärten. Ein Beispiel sind die Kennzahlensysteme zur Bewertung von Unternehmen. Obwohl von vielen Praktikern als Spielerei abgetan, ist die Kennzahlenanalyse ein wissenschaftlich hochaktuelles Thema. Insbesondere in der Verbindung von statistischen Methoden der Klassifikation und Diskriminanzanalyse mit den Methoden der Künstlichen Intelligenz zur Mustererkennung und Verwertung von Erfahrungswissen verspricht man sich wesentliche Fortschritte. Zur Kreditwürdigkeitsprüfung und Finanzberatung verwenden die deutschen Sparkassen schon seit einigen Jahren ein auf einem immensen Datenmaterial beruhendes, computerisiertes Auswertungssystem zur statistischen Bilanzanalyse (STABIL). Und gerade die gehobenen Unternehmensberatungen operieren mit Kennzahlen und Meßmethoden. Sie bilden deren substantielles Know-how.

Um nicht den Verdacht aufkommen zu lassen, daß das im obigen Argument erfolgte Herausstreichen der unternehmenspolitischen Aufgabenbereiche, wobei die Betonung auf dem Wort politisch liegt, ein Rückzugsgefecht auf eben diese Bereiche darstellt, wird man zweitens einwenden, daß die computergestützten Informationsund Planungssysteme im Augenblick nicht gut genug sind und auch nicht in ferner Zukunft den Qualitätsstandard erreichen, um in der oben angeführten Weise Kompetenzen vom Menschen auf die Maschine zu verlagern.

Zufriedenstellende Lösungen gibt es tatsächlich höchstens in Teilbereichen. Die Versuche, integrierte leistungsfähige Systeme zu schaffen, sind bisher gescheitert. Es ist aber gefährlich, diese Schwäche in die entferntere Zukunft hinein zu extrapolieren. Man muß sich bewußt sein, daß Software im Vergleich zum menschlichen Individuum nahezu unsterblich ist. Selbst der intelligenteste Mensch unterliegt dem Fluch des Alterns und Vergehens. Neue Generationen müssen beim Wissenserwerb stets bei Null anfangen. Dies gilt für Computersysteme nicht. So wie der technische und wissenschaftliche Fortschritt zunimmt, wächst auch die Leistungsfähigkeit derartiger Systeme. Die Rechner werden nie langsamer, sondern höchstens schneller und nach KARL POPPER setzen sich im Sinn eines Evolutionsprozesses langfristig auch die besseren wissenschaftlichen Theorien durch. Betriebliche Informations- und Planungssysteme, die auf wissenschaftlichen Methoden basieren, können also langfristig ebenfalls nicht schlechter, sondern eher besser werden.

Ist es also nur eine Frage der Zeit, bis die computergestützten betrieblichen Anwendungssysteme gut genug sind und vielleicht später den Menschen übertreffen? Im Sicherheitsbereich von Kernkraftwerken und bei der Steuerung von Großraumflugzeugen haben die Automaten den Handlungsspielraum des Menschen bereits wesentlich eingeengt. So sind heute automatische Maßnahmen zur Beherrschung des Störfalls vorgesehen, die das hohe Risiko durch menschliches Verhalten ausschließen sollen ([7]). 
Die Antwort lautet also: im Prinzip ja, falls menschliche Intelligenz und Computerintelligenz durch ein und dasselbe Maßsystem vollständig beschrieben werden können. (Letztendlich ist die entscheidende Frage, ob menschliche Intelligenz überhaupt vollständig in Maß und Zahl zu fassen ist. Denn dann ist jeder Zustand und jede Änderung als Punkt beziehungsweise als Abbildung in einem geeignet definierten mathematischen Raum zu beschreiben und kann auf einer Maschine prinzipiell nachgebildet werden.) Andernfalls lautet die Antwort: höchstens teilweise. Es gäbe dann auch bei noch so perfekten Anwendungssystemen immer noch Aufgabenbereiche, in denen das menschliche Vorgehen prinzipielle qualitative Unterschiede zum Handeln des Computers aufweist.

Hier werden Kernfragen des Menschseins berührt. Über das menschliche Gehirn und die sich in ihm vollziehenden Denkprozesse wissen wir noch sehr wenig ([8]). Neuronale Netze und Konnektionismus beschreiben nur die Verdrahtungsmechanismen und das Erkennen von Mustern. Die zerebrale Aktion muß jedoch in ein geistiges Erlebnis umgewandelt werden im Sinne von Merkmalsextraktionsleistungen. Die "Software" des Denkens ist noch weitgehend unerforscht.

Wenn es gelingt, eine Hardwarekopie des menschlichen Gehirns zu erstellen, kann der Rechner potentiell dieselben Leistungen erbringen wie das Gehirn. Dazu benötigt man aber noch die Software, denn sie ist nicht physikalisch realisiert. Die Frage „Wo ist der selbstbewußte Geist lokalisiert?" ist im Prinzip nicht zu beantworten ([8]). Bei der Erstellung der Software steht man vor unüberwindlichen Schranken. Um das menschliche Denken in ein Computerprogramm umzusetzen, muß es formalisierbar sein. Aufgrund der Erkenntnisse der Entscheidungstheorie wissen wir, daß jedes formale Denken auf nicht formalisierten Voraussetzungen beruht. Zwar kann man sie reflektieren und ihrerseits formalisieren, kontrollieren und korrigieren, aber nur um den Preis neuer, nicht formalisierter Grundannahmen. Es gibt keine verläßliche Methode, aus gegebenen Voraussetzungen jeden wahren Schluß zu ziehen. Eine vollständige formale Absicherung des Denkens mit den Mitteln des Denkens ist grundsätzlich unmöglich, das Denken kann sich nicht selbst vollständig begreifen ([2]).

Die Theologie gibt eine eindeutige Antwort: Zwar hat Gott in der Welt alles „geordnet mit Maß, Zahl und Gewicht" (Weisheit 11, 21 und Hiob 28, 25). Aber die Weisheit „ist das Hauchen der göttliche Kraft" (Weisheit 7, 25) und deshalb nicht materieller Natur. SALOMON "verglich ihr keinen Edelstein; denn alles Gold ist gegen sie wie geringer Sand" (Weisheit 7, 9).

\section{Die beiden Wissensquellen des Computers}

Es fällt leichter, zukünftige Entwicklungen abzuschätzen, wenn man den Wissensgenerierungsprozeß und den Interaktionsmechanismus Mensch - Maschine zu verstehen versucht. Die computergestützten Systeme machen sich zwei Wissensquellen zunutze. 
Die Informationsbasis im Unternehmen ist das Faktenwissen. Es besteht aus einem riesigen Zahlenfriedhof, der die Zustände und Änderungen an den Orten des Geschehens, das heißt zum weitaus überwiegenden Teil auf den unteren betrieblichen Ebenen beschreibt. Dieser Informationsbrei übersteigt das menschliche Fassungsvermögen. Die Information muß deshalb aufbereitet werden. Sie wird selektiert und verdichtet. Dies geschieht in der Regel in mehreren Zwischenstufen. Mit dem Selektions- und Verdichtungsprozeß geht aber stets ein Verlust an Information einher. Auch hier gilt das Gesetz, daß durch die Verarbeitung die Entropie zunimmt. Die Verarbeitungschritte sind nicht umkehrbar eindeutig. Nehmen wir als Beispiel die Deckungsbeitragsrechnung. Der Deckungsbeitrag $D B I$ (Nettoerlös minus variable Kosten) ist ein Durchschnittswert. Auf das einzelne Fertigteil bezogen ist er aller Wahrscheinlichkeit nach sogar falsch, denn die tatsächlichen variablen Kosten sind sehr stark von der augenblicklichen Situation abhängig. (So kann zum Beispiel das Rohmaterial für das gerade betrachtete Teil billiger als sonst sein, weil der Ausschußanteil in der letzten Anlieferung wesentlich niedriger als der kalkulierte Wert war.) Durch die Reduktion auf den Durchschnittswert gehen sämtliche Einzelinformationen verloren.

Nun ist es aber trotz Datenreduktion und damit Informationsverlust - zum Beispiel bei der Bildung von Durchschnittswerten - dennoch sehr aufschlußreich, statistische Kenngrößen zu verwenden. Nicht nur, weil sie übersichtlicher sind, sondern weil dadurch Information und auch Wissen einer neuen Qualität gegeben ist. Dies entsteht aber nicht aus dem Nichts, sondern stammt aus dem Wissen, auf das sich die Methoden gründen. Das Methodenwissen ist die zweite Wissensquelle.

So ist es möglich, zum Beispiel mittels statistischer Methoden Zusammenhänge herauszudestillieren, die bislang verborgen oder nur verschwommen bekannt waren. Korrelationen können auf Signifikanz getestet werden. In dem vorhin erwähnten Beispiel der Deckungsbeitragsrechnung wird durch die Bildung des Durchschnittswertes aus dem vorliegenden Faktenwissen neues Wissen induziert. Nicht der Durchschnittswert an sich ist die neue Information (im Gegenteil: die Gesamtheit der Einzelwerte birgt mehr Information als durch den Durchschnittswert repräsentiert wird). Neu ist das Wissen, daß es vernünftig ist, bei konstantem Bedingungsrahmen auch zukünftig Deckungsbeiträge in der Nähe dieses Wertes zu erwarten. Dieses Wissen um zukünftige Erwartungen geht weit über das Faktenwissen hinaus.

Dieses statistische Grundwissen ist nicht betriebsspezifisch. Es ist von seiner Natur her exogen und nur durch die Implementierung der Methode endogenisiert. Auf diese Weise erhält man Wissen in Form von Verfügungswissen, das heißt ein Wissen um Ursachen, Wirkungen und Mittel (wie die Dinge zusammenhängen, wie man sie beeinflussen kann, was zukünftig zu erwarten ist). Dieses zusätzlich geschaffene Wissenspotential dient dem Orientierungswissen (warum etwas der Fall ist, was (begründet) der Fall sein soll, und wohin die Reise gehen soll ([5])).

Ob durch die verwendete Methode tatsächlich Wissen induziert wird oder nur Irreführung, hängt von der Methode ab. Wissensgenerierend wirkt eine Methode nur dann, wenn sie schlüssig ist. Das ist sicher der Fall, wenn sie nur Schlußwei- 
sen der mathematischen Logik und statistische Regeln verwendet und sich auf das Axiomengebäude der Entscheidungstheorie gründet. Darüber hinaus stellt die Betriebswirtschaftslehre der Praxis laufend neue Instrumente zur Verfügung, in denen pragmatisches Wissen niedergelegt ist. In letzter Zeit gewinnt auch die Endogenisierung von Expertenwissen an Bedeutung. Durch den Einsatz von Expertensystemen verspricht man sich einen Quantensprung in der Leistungsfähigkeit.

Auch ist es im operativen Bereich längst üblich, Methodenwissen nicht nur zur Entscheidungsunterstützung einzusetzen, sondern Reaktionen auf bestimmte Systemzustände vom Menschen direkt auf die Maschine zu übertragen. Ein Beispiel liefert die petrochemische Industrie. Auf lokaler Ebene wird ein chemischer Produktionsprozeß automatisch gesteuert, in dem das Programm die Steuergrößen entlang der optimalen Trajektorien führt. Auf Konzernebene erfolgt routinemäßig die Produktionsplanung und Logistikplanung für Hunderte von Verarbeitungsanlagen und Hunderte von Zwischen- und Fertigprodukten.

\section{Stärken und Schwächen von Anwendungssystemen}

Faßt man das bisher Gesagte zusammen, kann man feststellen:

1. Es besteht nicht die Gefahr, daß ein Informationssystem aus sich heraus Wissen generiert. Es bleibt nur so schlau, wie es das endogenisierte Methodenwissen erlaubt.

2. Die Stärken liegen im operationalen Bereich. Dort lassen sich konkrete Aufgabenstellungen noch am ehesten ohne großen Realitätsverlust isolieren und als geschlossene Systeme behandeln, die mit der Umwelt nur in sehr geringem Umfang interagieren. Das vom Menschen in die wissenschaftlichen Methoden hineingedachte Wissen ist standardisiert und deshalb typischerweise für geschlossenen Systeme geeignet. Für offene Systeme ist diese Wissensquelle unzureichend.

3. Die Unternehmensführung verlangt sehr viel exogenes Wissen und eine intensive Interaktion mit der Außenwelt. Ein Unternehmen ist ein hochgradig komplexes offenes System. Hier ist der Mensch als Individuum mit seinem Wissen und seiner Genialität unersetzlich. Die Führungsinformationssysteme nehmen letztendlich nur eine Umformung der Basisinformation in ein Muster vor, das geignet ist, vom menschlichen Geist erfaßt und verarbeitet zu werden. Er durchschaut besser, was ihm an Information zur Verfügung steht. Die Verarbeitung geschieht dadurch, daß dieses Muster am Erfahrungschatz des Menschen reflektiert wird und dort die eigentlichen Denkprozesse in Gang setzt. 
Die Vorstandspositionen bleiben also auf Sicht ungefährdet. Anstatt in Konfrontation zu treten, ist es vernünftiger, die Betriebsinformatik weiterzuentwickeln. Dies soll in erster Linie dort geschehen, wo sie ihre typischen Stärken ausspielen kann: im Methodenbereich.

\section{$5 \quad$ Beseitigung des Methodendefizits}

An der Verbesserung der Informationsbeschaffung und -verwaltung (Datenmenge, Zugriffsgeschwindigkeit, Datenqualität) wird laufend gearbeitet, unter anderem im Rahmen des Computer Integrated Manufacturing.

Anders ist es beim Methodenwissen. Obwohl in ihnen die qualitativ höherwertigen Wissensanteile stecken, vollziehen sich Methodeninnovationen mit beträchtlicher Zeitverzögerung. Der wissenschaftliche Fortschritt erfährt keine permanente Umsetzung in die Anwendungssysteme. Dies hängt sehr eng mit dem Ausbreitungskonzept der Datenverarbeitung im Unternehmen zusammen. Es setzt voraus, daß der DVEinsatz auf einer höheren Unternehmensebene sich auf eine solide DV-Basis der nächstniedrigeren Ebene stützen kann. Man will an den tieferliegenden Schichten wenig ändern, bevor nicht das Gesamtgebäude abgeschlossen ist. Dies trifft sowohl für Unternehmen zu, die ihrer Informatikstrategie ein langfristiges Konzept zugrunde gelegt haben, als auch Unternehmen, bei denen die betriebliche Datenverarbeitung historisch gewachsen ist. Änderungen sind in jedem Fall mit hohen Kosten verbunden.

Eine zweite Ursache für das vorliegende Methodendefizit ist auch darin zu sehen, daß bei der Systementwicklung das Augenmerk des Anwenders zu sehr auf das Was und zu wenig auf das Wie gerichtet war. Die Methodenauswahl überließ man häufig dem Softwareentwickler. Dessen Know-how liegt aber auf dem Gebiet der Softwareproduktion und weniger bei den betriebswirtschaftlichen Instrumenten. So kam es, daß zu sehr die personellen Techniken kopiert wurden ([9]). Man macht es manches Mal im Prinzip so, wie man es bereits früher gemacht hat; nur jetzt schneller, weil auf dem Computer.

Vor zwanzig Jahren stand die Unternehmensforschung an einem Scheideweg. Der Technologietransfer fand nicht statt. Sogar der Kommunikationsprozeß zwischen Theoretikern und Praktikern war so spärlich ausgebildet, daß die Entwicklung zu theorielastig wurde und andere in die Bresche springen mußten. Heute, wo sich mit der künstlichen Intelligenz ein mindestens ebenso bedeutsames Feld auftut, steht nach Peter Mertens ([4]) die Expertensystem-Forschung an einer ähnlichen Weggabelung.

Mit zunehmendem Time-lag und wachsender Konkurrenz wird jedoch der Druck zur Beseitigung des Methodendefizits immer stärker. 


\section{Zielsetzung Basisprobleme}

Eine differenziertere Betrachtung legt den Schluß nahe, Methodeninnovationen nicht in der mittleren beziehungsweise oberen Unternehmensschicht durchzuführen, sondern im Basisbereich. Zum einen sind dort die Kostenbarrieren niedrig, zum anderen sind die unten anzutreffenden Probleme am ehesten von der Art geschlossener Systeme. Zum dritten, und hier muß ein neues Problembewußtsein geschaffen werden, ist es gut, sich auf die Probleme an der Basis zu besinnen, denn dort entstehen die wesentlichen Kosten und dort können sie am effektivsten beeinflußt werden. Die Japaner haben diese Innovationslücke bereits erkannt. Sie scheuen sich nicht, computergestützte betriebswirtschaftliche Instrumentarien für Basisprobleme auf einem höchst anspruchsvollem Niveau in der Praxis einzusetzen. Auch in den USA ist man auf diesem Weg ([11]).

Anhand von zwei Kooperationsprojekten soll nun gezeigt werden, daß durch die Methodeninnovationen auf der unteren Unternehmensebene nicht nur marginale Verbesserungen zu erzielen sind, sondern erhebliche Optimierungsreserven ausgeschöpft werden können, die durchaus für das gesamte Unternehmen von Bedeutung sind.

Das erste Projekt ist eine Fallstudie aus der Konsumgüterindustrie. Das betreffende Unternehmen besitzt in der Bundesrepublik Deutschland zwei Produktionsstätten, an denen schnell verderbliche Güter hergestellt werden. Die Frische der Ware ist das wichtigste Verkaufsargument. Sofort nach der Herstellung werden die Artikel auf Lastwagen verladen und in insgesamt fünf Regionalläger transportiert. Von dort wird das Filialnetz, bestehend aus mehreren tausend Verkaufsstellen, in täglicher Belieferung bedient.

Ziel der Untersuchung war die Entwicklung von Strategien zur Reduktion der Lagerverweilzeiten, der Bestände und der Logistikkosten.

Es hat sich gezeigt, daß durch die Errichtung eines Informationssystems und die Verwendung von wissenschaftlich zwar anspruchsvollen, aber in der Handhabung einfachen Methoden auf unterster Ebene, nämlich bei den Verkaufsstellen, ein enormer Effekt zu erreichen ist. Mit Hilfe einer tagesgenauen Absatzprognose für jeden Artikel und feinstrukturierter Bestellregeln konnten zwei Ziele alternativ erreicht werden:

1. Bei unverändertem Belieferungsplan konnte ein Frischegewinn von insgesamt $20 \%$ realisiert werden.

2. Bei unveränderter Frische und unveränderten Durchschnittsbeständen ist eine Belieferung nur dreimal pro Woche (Montag, Mittwoch, Freitag) ausreichend. Dies bringt eine Halbierung der Transportkosten mit sich. Dieser Einsparungseffekt auf unterster Ebene pflanzt sich verstärkt auf höherer Ebene fort. Es ergeben sich Konsequenzen bezüglich der Regionallagerstruktur und insgesamt eine Neuorganisation der gesamten Distributionslogistik. Das Einsparungspotential beträgt weit über 1 Mio DM pro Jahr. 


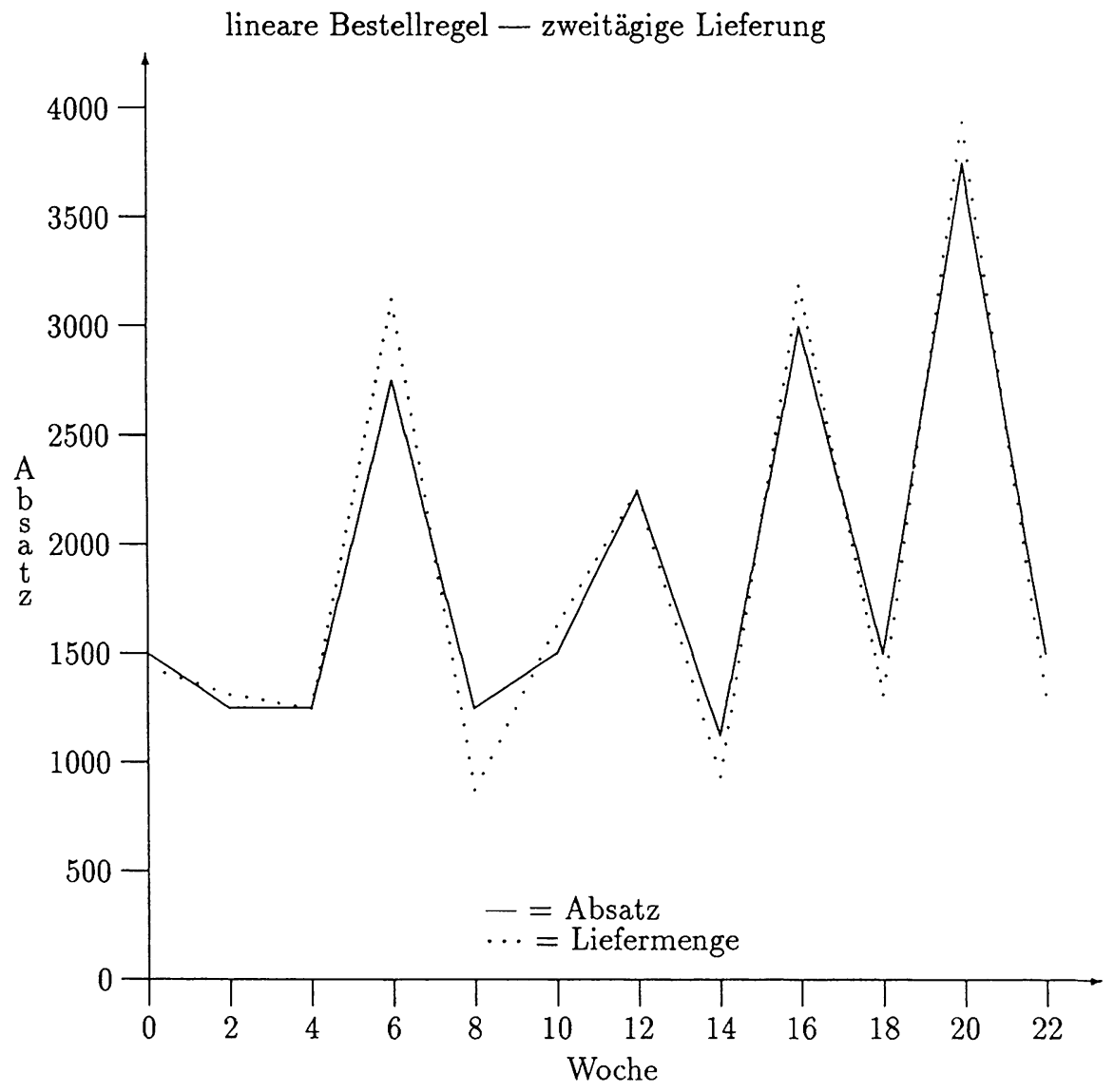

Abbildung 1: Absatz versus Liefermenge 
In diesem Fall geschieht der Wissenszuwachs durch Prognosen und Steuerung der Risikobestände mit Hilfe von Methoden aus der Entscheidungstheorie. Die Abbildung 1 zeigt, wie eng die Liefermengen an den tatsächlichen Bedarf herangeführt werden konnten. Die Sicherheitsbestände wurden um sämtliche überflüssigen Reserven reduziert.

Das zweite Beispiel betrifft die Papierherstellung. Die papiererzeugende Industrie sieht sich vor große Herausforderungen gestellt:

- ein sich verändernder Markt (wachsende Marktmacht der Großkunden, schwindende Kundentreue, große Nachfrage),

- Umweltinvestitionen zur Reinhaltung der Gewässer,

- sparsamer Umgang mit Rohmaterial und Energie.

Die sich zum Teil widerstrebenden Ziele der kundennahen Fertigung mit sehr kurzen Lieferzeiten und Produktionsflexibilität zum einem, der festgefügten optimierten Arbeitsabläufe mit hoher Maschinenauslastung zum anderen sowie der Minimierung des Einsatzes von Energie- und Materialressourcen verlangen moderne DV-Lösungen im Planungs- und Steuerungsbereich.

Für die Optimierung auf Werkstattebene, insbesondere der Zuschnittsplanung und der Maschinenbelegungsplanung stehen DV-Verfahren zur Verfügung. Wegen der mathematischen Komplexität der Probleme sind die Verfahren nur eingeschränkt geeignet. Im allgemeinen sind sie gegenüber einem erfahrenen Handplaner nicht konkurrenzfähig. Die Fähigkeit des Menschen, ähnlich wie beim Schachspiel eine Situation sehr schnell zu überblicken und günstige Lösungen zu erkennen, ist beachtlich. Die naive Methode des Kopierens der menschlichen Vorgehensweise scheitert daran, daß es keine einfachen Kochrezepte gibt.

Erst die sorgfältige Analyse des Erfahrungswissens guter Handplaner brachte den Durchbruch. Ein wissensbasierter Ansatz in Verbindung mit speziellen Optimierungsverfahren führte zu einem Quantensprung in der Leistungsfähigkeit. In Kooperation mit fünf großen deutschen Papierhersteller wurde ein wissensbasiertes System geschaffen, das in aller Regel die Zielsetzungen Durchsatzmaximierung, Verschnittminimierung und Bestandsoptimierung wesentlich besser als der Mensch löst.

Der durch eine nicht optimale Verkombinierung verursachte sogenannte planerische Verschnitt liegt zwischen $2 \%$ und $5 \%$ der Gesamtproduktion. Mit Hilfe des Computersystems wird er auf $0,5 \%$ bis $2,5 \%$ gedrückt. Das bedeutet, daß durch diese Maßnahme auf einer sehr tief angesiedelten Ebene die Umsatzrendite des Unternehmens um bis zu 2 Prozentpunkte gesteigert werden konnte.

\section{Konklusion}

Eine Verweigerungshaltung der Unternehmensführung gegenüber modernen computergestützten betrieblichen Führungsinstrumenten wäre irrational und unbegründet. Es ist jedoch wichtig, sich beim Blick in die Zukunft nicht nur auf die enorm kapitalintensiven Informatikkonzepte der Factory of the Future und auf die DV im 
strategischen Management zu konzentrieren, sondern auch die innerbetrieblichen Methodendefizite zu erkennen und durch gezielte Methodeninnovation auf operationaler Ebene zu schließen. Zukunftsweisende Perspektiven erhalten ihre auslösenden Impulse (und auch das notwendige Kapital zu ihrer Verwirklichung) von originellen Lösungen an der Basis.

\section{Literatur}

[1] von Bechtolsheim, Matthias; Musshoff, H. Josef: Falsche Vorstellungen revidieren - MIS im zweiten Frühling, in: Computerwoche Extra 3 (1989).

[2] Gierer, Alfred: Die Physik, das Leben und die Seele - Anspruch und Grenzen der Naturwissenschaft, München 1985.

[3] Mertens, Peter; Plattrault, Eberhard: Informationstechnik als strategische Waffe, in: Information Management 2 (1986), 6 - 17.

[4] Mertens, Peter: Knochenarbeit geleistet, in: Computerwoche, vom 29. Januar 1988.

[5] Mittelstrass, Jürgen: Computer und die Zukunft des Denkens, Vortragsmanuskript, Universität Konstanz, 19. Mai 1989.

[6] o. V.: Sonderauswertung Statistische Bilanzanalyse (STABIL) für EBIL-Bilanzen, in: BSGV-Mitteilung Nr. 145 von 23. Mai 1986.

[7] PaUl, GÜNTER: Reaktorsicherheit zuversichtlicher beurteilt - Ergebnisse der Deutschen Risikostudie Kernkraftwerke (Phase B), in: Frankfurter Allgemeine Zeitung Nr. 152, 1989.

[8] Popper, Karl R.; Eccles, John C.: Das Ich und sein Gehirn, München, Zürich 1982.

[9] Scheel, Joachim: Nachdenken statt nachmachen, in: Computerwoche Extra 3 (1989).

[10] Zuboff, SнолнANa: In the age of the smart machine: the future of work and power, Basic Books Inc., New York 1988.

[11] Zuboff, Shoshana: IT and authority: the case of Tiger Creek mill, in: The McKinsey Quarterly Winter 1989. 